\title{
Physical activity classification using a smart textile
}

\author{
Nour Cherif*†, Youssef Ouakrim*, Amel Benazza-Benyahia ${ }^{\ddagger}$, Neila Mezghani* \\ ${ }^{*}$ Laboratoire de recherche en Imagerie et orthopédie (LIO), Centre de Recherche LICEF, TELUQ university, Montreal, Canada \\ ${ }^{\dagger}$ Faculty of Science and Technology, University of Lorraine, France \\ ${ }^{\ddagger}$ COSIM Lab, École Supérieur de communication de Tunis (Sup’Com), Tunis, Tunisia
}

\begin{abstract}
The aim of this study is to develop a human activity classification system based on a wearable intelligent textile and machine learning techniques. Using the Relief-F feature selection algorithm, we identified a set of relevant features collected by the smart textile. Then, the retained features have fed a classifier in order to recognize the underlying activity. In this respect, we test a support vector machine classifier (SVM) and a $k$-nearest neighbor classifier (KNN). The results show the reliability of the feature selection procedure and indicate that the activities can be recognized with an overall accuracy of more than $96.37 \%$ using the KNN classifier and $95.4 \%$ using the SVM classifier. Since the Hexoskin intelligent textile also allows the collection of physiological data, these experimental results are very promising for practical applications of acquisition of human activities recognition, which will make it possible to study the patient's state of health or to detect physiological abnormalities in real time depending on the physical activity exerted.

Index Terms-Wearable sensors, human activity classification, feature selection, triaxial accelerometer, physical activity.
\end{abstract}

\section{INTRODUCTION}

The automatic classification of physical activity using wearable sensors is trending among the areas of research for realtime monitoring of human movements. This can provide an automated system to monitor physiological functions over long periods. In this study, we are using the Hexoskin intelligent textile ${ }^{1}$, developed by Carré technologies (Montréal, Canada) which is an easy to put on and comfortable stretch shirt that can be used in any ambient environment. The Hexoskin data acquisition is non-invasive and can be performed continuously without hampering the movements of the person wearing it. In practice, Hexoskin enables real-time remote monitoring of $3 \mathrm{D}$ acceleration data, cardiac activity and respiratory activity. Furthermore, through Bluetooth technology, the acquired data can be easily transferred to a smart device in order to be remotely analyzed. At this level, it is worth noting that for several applications such as health, safety and sport, it is highly desirable to design an automated and personalized physical activity monitoring system operating in real time at a low cost price. For instance, physiological data (cardiac and breathing data) are of interest since they allow the caregivers to study the state of wearer health and to detect physiological abnormalities in real time according to the physical activity carried out.

In this paper, we focus on activity monitoring based on data acquired by the Hexoskin textile. Our work consists in designing a real-time feature-based procedure for activity

${ }^{1}$ http://www.hexoskin.com/ recognition: features are extracted, selected, and then they feed a classification algorithm to output the type of physical activity. Our contributions are to tackle the delicate problem of selecting the most relevant features and to develop a robust classification system to recognize in real time the physical activity.

This paper is organized as follows. In Section II, we give an overview of the reported research on activity recognition from body-worn sensors. In Section III, we present the proposed operational method. Section IV is dedicated to the experimental results. Finally, in Section V, some conclusions are drawn.

\section{RELATED WORKS}

Some of the activity recognition works focus on the use of one or multiple accelerometers and possibly other sensors. In [1], an overview of related works in human activity recognition is performed. For each method, the authors specify the underlying activities, the types of sensors, the extracted features (and, possibly, the feature selection strategy), the classification method applied, and the achieved accuracy. Similarly, in [2], the authors give a summary of the different techniques that were used to classify normal activities and/or identify falls from body-worn sensor data. This is also the case in the work of Mannini and Sabatini [3] concerning data involving accelerometers. In their review, the best classification rate is of $98.8 \%$ and, it is obtained with a wavelet-based features and threshold classifiers [4]. Note that the data were collected from a 3D accelerometer from 20 subjects to distinguish walking on level ground from walking on a stairway. From these overviews, it can be noted that the activity recognition procedure could be decomposed into three main steps: the computation of features, the selection of relevant features and, the classification guided by the retained features.

Several activity classification studies have used a wide range of approaches to compute the features. They can be divided according to the domain from where the features are extracted [5]. Time-domain are typically statistical measures including the mean, median, variance or standard deviation, skewness, kurtosis, energy and correlation between axes [1]-[3], [6]-[8]. Activity descriptors can also be computed in the frequency domain such as the Fast Fourier Transform (FFT) [9] or the discrete cosine transform coefficients [10].

Once the features are generated, the second step is to apply a feature selection method to the feature set to find the most salient descriptors able to increase the accuracy of the 
classification and its computational load. In general, feature selection techniques can be grouped into three categories: filter methods, wrapper methods and embedded methods [11]. Filter based feature selection methods are generally faster than wrapper based ones. A study of feature selection methods in general as well as in the field of human activity recognition can be found in [2], [12]-[14]. Then, the retained features are used as inputs in a classification algorithm. In [2], the authors give a state of the art of the different classification techniques taken from the machine learning field.

It is also worth mentioning that the number and the type of sensor accounts for the achieved performances. For example, in [17], the authors used 5 accelerometers worn on different parts of the body to recognize user physical activity such as walking, sitting, watching $\mathrm{TV}$, running, eating and reading. The overall accuracy was $84 \%$. In another study [18], the authors even used a total of 30 sensors that were embedded in a garment and spread across the body to improve recognition rates for a relatively complex set of activities. However, increasing the number of sensors could lead to the risk that the users could feel uncomfortable with many sensors, as they could prevent them from doing their activities in a natural way. In this respect, a lot of attention should be given to a smart textile with an acceptable amount of light sensors (such as the Hexoskin). In a recent paper [15], a database related to Hexoskin textile was used only for the detection of falls and their orientations. The falls were classified according to their orientations among 11 classes of physical activities. The fall detection system reaches the accuracy of $98 \%$. In this work, we tackle a more challenging context. Indeed, we consider only one accelerometer embedded at the waist level in the textile. The classification deals with a high number of activities (11 activities) including an unknown activity or null activity class (any activities out of the activities of interest). Moreover, the developed classification system operates in real time.

\section{METHOD}

Our objective is to recognize the ten physical activities and also the unknown activities (transition class) from the acquired data from the 3 -axis accelerometer. The block diagram of the proposed method is described in Fig. 1.

\section{A. Peak Detection and Windowing}

The sequence of the norm of the acceleration vector is firstly computed and divided into short time windows by detecting peaks in real time. More precisely, peaks are detected by thresholding the norm. The value of the threshold was adjusted empirically to 1.1 . Then, a rectangular window of 90 points around each detected peak is identified so as its temporal duration is around $1 \mathrm{~s}$. For each window, several features are computed to characterize the signal.

\section{B. Feature extraction}

We recall that features are values or vectors that represent the relations of the data in some descriptive and discriminating

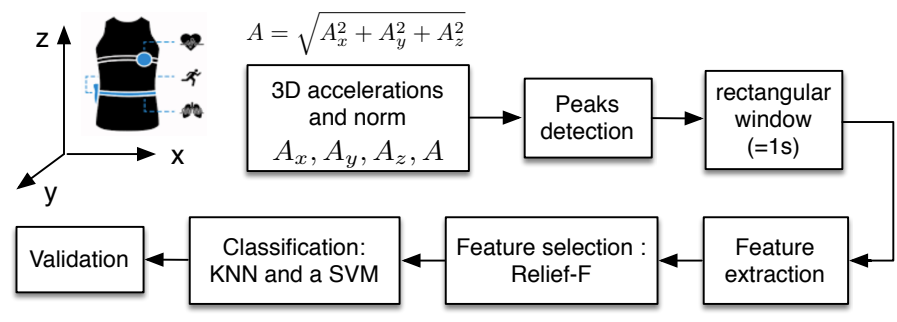

Fig. 1. Block diagram of the proposed Physical activity classification system.

way [19]. Let $A_{x}, A_{y}$ and, $A_{z}$ the accelerations along respectively the $x, y$ and, $z$ axes. The norm $A$ of the acceleration vector is defined by:

$$
A=\sqrt{A_{x}^{2}+A_{y}^{2}+A_{z}^{2}} .
$$

For the sake of simplicity, let $S_{n}$ denote either of the component $A_{x}, A_{y}, A_{z}$ or $A$ at time $n$.

For each $S_{n}$, the following time-domain features have been considered in each window, minimum, maximum, mean, variance (VAR), standard deviation (STD), kurtosis, skewness, energy, Root Mean Square (RMS), Zero Crossing Rate (ZCR), Euclidean norm, Maximum Absolute Column Sum (1-Norm), amplitude and correlation. The frequency domain feature used is the maximum magnitude of the Fast Fourier Transform (FFT). Also, some conventional statistical features are used such as the simple correlation value (Cor).

\section{Feature Selection Using Relief-F}

The Relief-F algorithm was used to identify the most important features [21]. The Relief-F algorithm is one of the popular filters based feature selection methods to estimate the weight of features. It can efficiently estimate the quality of features in classification problems with strong dependencies between features which is an important aspect in our case. The algorithm ranks individual features according to feature relevance scores. It randomly selects an instance $R_{i}$ then finds the $k$ nearest neighbors from the same classes, called nearest hits $H_{j}$ and the $k$ nearest neighbors from the different classes, called nearest misses $M_{j}(C)$. It updates the quality estimate $W[A]$ for all features $A$ according to their values for $R_{i}, H_{j}$ and $M_{j}(C)$. Instead of finding a single miss from a different class, Relief-F finds one near miss $M_{j}(C)$ for each different class $C$ and averages their contribution for updating the estimate $W[A]$. The average is weighted with the prior probability of each class $P(C)$. The process is repeated for $m$ times. User-defined parameter $k$ controls the locality of the estimates. $\operatorname{dif} f(A, I 1, I 2)$ calculates the difference between the values of the attribute $A$ for two instances $I 1$ and $I 2$. Function $\operatorname{diff}$ is also used for calculating the distance between instances to find the nearest neighbors. The whole distance is simply the sum of differences over all attributes. A more detailed discussion can be found in [22]. 


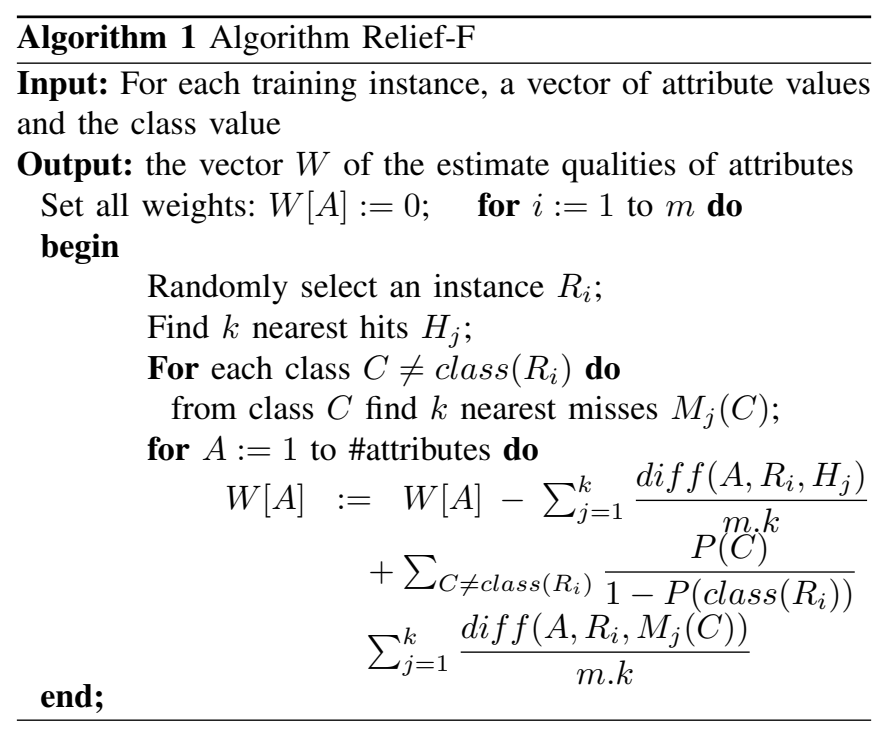

\section{Classification}

The retained features serve the classification algorithm. We investigated two classification methods: (1) A K Nearest Neighbor (KNN) which is a non-parametric supervised method [29]. A given observation is assigned to the class to which the majority of its $k$ nearest neighbors of the training data set belong and (2) A support vector machine (SVM) which is a supervised machine learning algorithm that aims to find the optimal separating decision hyperplanes between classes with the maximum margin between patterns of each class. The classifier performances are evaluated in terms of classification accuracy and classification accuracies per class. For this purpose, the database is randomly divided into three sets: a training set, a validation set and a test set.

\section{EXPERIMENTAL RESULTS}

\section{A. Data collection}

Thirteen healthy volunteers (age $25.43 \pm 7.51$ years old, weight $60.7 \pm 6.7 \mathrm{~kg}$, heigh $172.7 \pm 7.2 \mathrm{~cm}$ ) have participated to this study. The data collection was firstly approved by institutional ethics committees, with all subjects providing written informed consent before their participation. Data collection was performed at the research center of the Hospital Center of Montreal University (Canada). Each participant wore the smart textile Hexoskin and repeated 5 times a sequence of 10 tasks such as going up the stairs, going down the stairs, walking, running, sitting, fall right, fall left, fall backward, fall forward and lying. The 11th task corresponds to unknown activities. The accelerations were collected from the 3-axis sensors integrated in the Hexoskin with a 13-bit resolution and a frequency of $64 \mathrm{~Hz}$.

\section{B. Peak Detection and Feature Extraction}

As mentioned in the previous section, in order to segment the signals into windows, real-time detection of peaks is performed on the signal norm $A$. Once a peak detected, a rectangular window of one second was identified for feature extraction and classification. The total number of 58 features was extracted from $A_{x}, A_{y}, A_{z}$ and $A$. Fig. 2 displays the signal norm of the sequence of the ten performed tasks.

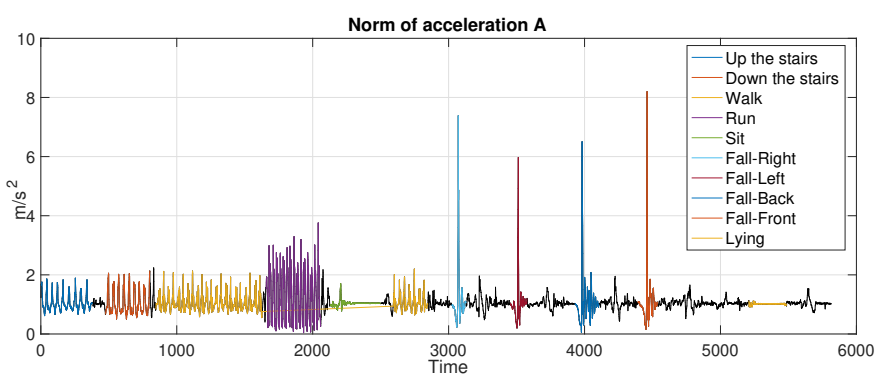

Fig. 2. Signal norm of the sequence of the ten activities.

\section{Feature selection and classification}

Physical activity classification system was developed using Matlab R2017a software (Mathworks, Massachusetts, United States). The system was developed using a machine Core i7920 with Intel-Core CPU $(2.67 \mathrm{GHz})$ and $12 \mathrm{G}$ of memory.

We investigated two classification methods, i.e., a KNN and a SVM classifier. The database was randomly divided into three sets: a training set ( $70 \%$ of the whole samples), a validation set $(15 \%)$ and a test set $(15 \%)$. To determine the best number $N$ of features, a 100 -fold cross-validation was performed. The $N$ features have been selected using the training set to produce the model. A set of 35 features was retained for the KNN and a set of 38 for the SVM. The selected features are then tested on an independent test set, giving $96.37 \%$ of accuracy using a KNN and $95.40 \%$ of accuracy when using an SVM (Table II). The retained features for the KNN are summarized in Table I, those for the SVM are slightly different.

TABLE I

THE 35 RETAINED FEATURES USING THE RELIEF-F ALGORITHM AND THE KNN CLASSIFIER.

Min $A$, ZCR $\left(A_{x}\right)$, MaxFFT $(A)$, Cor $\left(A_{x}, A_{y}\right)$, Cor $\left(A_{x}, A_{z}\right), \operatorname{ZCR}\left(A_{z}\right)$, Skewness $\left(A_{z}\right)$, Skewness $\left(A_{x}\right)$ Cor $\left(A_{y}, A_{z}\right)$, Skewness $\left(A_{y}\right)$, Skewness $(A)$, Kurtosis $(Z)$, Kurtosis $(A)$, max-min $\left(A_{y}\right)$, Kurtosis $\left(A_{x}\right)$ Kurtosis $\left(A_{y}\right)$, max-min $\left(A_{x}\right)$, STD $(A)$, Mean $\left(A_{z}\right)$, Min $\left(A_{y}\right)$, Mean $\left(A_{x}\right)$, STD $\left(A_{y}\right)$, MaxFFT $\left(A_{y}\right), \operatorname{Min}\left(A_{x}\right), \operatorname{Min}\left(A_{z}\right), \max -\min \left(A_{z}\right), \operatorname{STD}\left(A_{x}\right)$, Norm1 $\left(A_{x}\right), \operatorname{RMS}\left(A_{x}\right), \operatorname{Norm}\left(A_{x}\right), \operatorname{STD}\left(A_{z}\right)$, Mean $(A), \operatorname{RMS}\left(A_{z}\right)$ $\operatorname{Norm}\left(A_{z}\right)$, Var $(A), \operatorname{Norm} 1\left(A_{z}\right), \operatorname{MaxFFT}\left(A_{x}\right), \operatorname{Norm} 1(A)$

The classification accuracies per class (of different activities) on the test set are summarized in Table II. The most misclassified activity is the lying class. This is due to the small number of data in this class compared to other classes. Overall, with an accuracy of $96.37 \%$, KNN performs slightly better than SVM with $95.4 \%$ of accuracy. Using a KNN, Fall-Right and going Up the stairs have the highest recognition rate with an accuracy more than $98 \%$. 
TABLE II

ClAsSIFIER PERFORMANCE : ACCURACY (IN \%) AND ACCURACIES PER CLASS (IN \%)

\begin{tabular}{|c|c|c|}
\hline Classifier & KNN & SVM \\
\hline Number of features & 35 & 38 \\
Accuracy & 96.37 & 95.40 \\
\hline \hline Activities & KNN & SVM \\
\hline Up the stairs & 98.41 & 96.30 \\
Down the stairs & 94.97 & 95.53 \\
Walk & 97.44 & 97.85 \\
Run & 97.44 & 97.15 \\
Sit & 91.66 & 91.67 \\
Fall-Right & 98.76 & 90.12 \\
Fall-Left & 95.08 & 93.44 \\
Fall-Back & 91.78 & 89.04 \\
Fall-Front & 91.35 & 92.59 \\
Lying & 83.33 & 66.67 \\
Unknown & 96.24 & 94.97 \\
\hline \hline
\end{tabular}

\section{CONCLUSIONS}

In this study, a real-time activity classification system is presented. The system is based on smart textile and machine learning techniques. The feature selection was performed using the Relief-F algorithm which ranks individual features according to their relevance scores. The classification used the KNN and the SVM classifiers. Their performances have been tested on a high number of activities showing the reliability of the approach. The classification of physical activities is very important. Indeed, it offers opportunities to develop automated systems to monitor real-time changes in physiological data according the physical activities. Thus, it allows caregivers to study human health status and to detect physiological abnormalities in real-time depending on physical activities.

\section{REFERENCES}

[1] R. Damaševičius, M. Vasiljevas, J. Šalkevičius, and M. Woźniak, "Human activity recognition in all environments using random projections," Computational and Mathematical Methods in Medicine, vol. 2016, Article ID 4073584, 17 pages, 2016

[2] S. J. Preece, J. Y.Goulermas, L. P. Kenney, D. Howard, K. Meijer, and R. Crompton, "Activity identification using body-mounted sensors: a review of classification techniques," Physio. measurement, vol. 30, April 2009.

[3] A.Mannini and A. M. Sabatini, "Machine learning methods for classifying human physical activity from on-body accelerometers," Sensors, vol. 10, pp. 1154-1175, 2010.

[4] M. Sekine, T. Tamura, T. Togawa, and Y. Fukui, "Classification of waistacceleration signals in a continuous walking record," Medical Eng. \& Physics, vol. 22, pp. 285-291, 2000.

[5] D. Figo, P. C. Diniz, D. R. Ferreira, and J. M. Cardoso, "Preprocessing techniques for context recognition from accelerometer data," Personal and Ubiquitous Computing, vol. 14, pp. 645-662, 2010.

[6] J. Baek, G. Lee, W. Park, and B. J. Yun, "Accelerometer signal processing for user activity detection," Knowledge-Based Intelligent Information and Engineering Systems, Springer: Berlin, Heidelberg, pp. 610-617, 2004

[7] N. Ravi, N. Dandekar, P. Mysore, and M. L. Littman, "Activity recognition from accelerometer data," Conf. on Innovative applications of artificial intelligence, Pennsylvania, USA, pp. 1541-1546, 2005.

[8] S. Wang, J. Yang, N. Chen, X. Chen, and Q. Zhang, "Human activity recognition with user-free accelerometers in the sensor networks," Int. Conf. on Neural Networks and Brain, pp. 1212-1217, 2005.

[9] Y. P. Chen, J. Y. Yang, S. N. Liou, G. Y. Lee, and J.-S. Wang, "Online classifier construction algorithm for human activity detection using a triaxial accelerometer," Applied Mathematics and Computation, vol. 205, pp. $849-860,2008$
[10] Z. He and L. Jin, "Activity recognition from acceleration data based on discrete cosine transform and SVM," IEEE Int. Conf. on Systems, Man and Cybernetics, San Antonio, TX, pp. 5041-5044, 2009.

[11] Y. Saeys, I. Inza, and P. Larrañaga, "A review of feature selection techniques in bioinformatics," Bioinformatics, vol. 23, pp. 2507-2517, 2007.

[12] I. Guyon and A. Elisseeff, "An Introduction to variable and feature selection,” J. Mach. Learn. Res., vol. 3, pp. 1157-1182, 2003.

[13] H. Mazaar, E. Emary, and H. Onsi, "Evaluation of feature selection on human activity recognition," IEEE Int. Conf. on Intelligent Computing and Information Systems Cairo, Egypt, pp. 591-599, 2015.

[14] S. González, J. Sedano, J. R. Villar, E. Corchado, A. Herrero, and B. Baruque, "Features and models for human activity recognition," Neurocomputing, vol. 167, pp. 52-60 2015.

[15] N. Mezghani, Y. Ouakrim, M. R. Islam, R. Yared, and B. Abdulrazak, "Context aware adaptable approach for fall detection bases on smart textile," EEE Int. Conf. on Biomedical Health Informatics, Orlando, FL, USA, pp. 473-476, 2017.

[16] J. R. Kwapisz, G. M. Weiss, and S. A. Moore, "Activity recognition using cell phone accelerometers," SIGKDD Explor. Newsl., vol. 12, pp. 74-82, 2011.

[17] L. Bao and S.S. Intille, "Activity recognition from user-annotated acceleration data," Pervasive Computing Conf. Linz/Vienna, Austria, pp. $1-17,2004$.

[18] K. Van Laerhoven, A. Schmidt, and H. W. Gellersen, "Multi-sensor context aware clothing," International Symposium on Wearable Computers, Seattle, WA, USA, pp. 49-56 2002.

[19] D. Pyle, Data Preparation for Data Mining, Diane D. Ed.; Morgan Kaufmann Inc.: San Francisco, CA, 1999.

[20] K. Kira and L. A. Rendell, "The feature selection problem: traditional methods and a new algorithm," Conf. on Artificial intelligence, San Jose, California, pp.129-134, 1992.

[21] I. Kononenko, E. Simec, and M. Robnik-Šikonja, "Overcoming the myopia of inductive learning algorithms with relief," Applied Intelligence, vol. 7, pp. 39-55 1997.

[22] M. Robnik-Šikonja, and I. Kononenko, "Theoretical and empirical analysis of ReliefF and RReliefF," Machine learning, vol. 53, pp. 23-69, 2003.

[23] S. J. Preece, J. Y. Goulermas, L. P. Kenney, and D. Howard, "A comparison of feature extraction methods for the classification of dynamic activities from accelerometer data," IEEE Trans. on Biomedical Engineering, vol. 56, pp. 871-879, 2009.

[24] U. Maurer, A. Rowe, A. Smailagic, and D. Siewiorek, "Location and activity recognition using ewatch: A wearable sensor platform," Ambient Intelligence in Everyday Life, pp. 86-102, 2006.

[25] W. H. Wu, A. A. T. Bui, M. A. Batalin, D. Liu, and W. J. Kaiser, "Incremental diagnosis method for intelligent wearable sensor systems," IEEE Trans. on Information Technology in Biomedicine, vol. 11, pp. 553-562, 2007

[26] T. Huynh, and B. Schiele, "Towards less supervision in activity recognition from wearable sensors," IEEE Int. Symp. on Wearable Computers, Montreux, Switzerland, pp. 3-10, 2006.

[27] M. Ermes, J. Pärkkä, J. Mäntyjärvi, and I. Korhonen, "Detection of daily activities and sports with wearable sensors in controlled and uncontrolled conditions.," IEEE Trans. on Information Technology in Biomedicine, vol. 12, pp. 20-26, 2008.

[28] J. Parkka, M. Ermes, P. Korpipaa, J. Mantyjarvi, J. Peltola, and I. Korhonen, "Activity classification using realistic data from wearable sensors," IEEE Tran. on Information Technology in Biomedicine, vol. 10, pp. 119-128, 2006.

[29] P. Indyk, and R. Motwani, "Approximate nearest neighbors: towards removing the curse of dimensionality," ACM Symp. on Theory of computing, Dallas, Texas, USA, pp. 604-613, 1998.

[30] P. Domingos, and M. Pazzani, "On the optimality of the simple Bayesian classifier under zero-one loss,” Machine Learning, vol. 29, pp. 103-137, 1997.

[31] P.E. Utgoff, "Incremental induction of decision trees," Machine Learning, vol. 4, pp. 161-186, 1989.

[32] D. Michie, D. J. Spiegelhalter, C. C. Taylor, and J. Campbell, Machine Learning, Neural and Statistical Classification, Ellis Horwood: Upper Saddle River, NJ, USA, 1994. 